\title{
PROTEIN COMPOSITION CORRELATES WITH THE MECHANICAL PROPERTIES OF SPIDER (ARGIOPE TRIFASCIATA) DRAGLINE SILK
}

A Thesis

Presented to

The Graduate Faculty of The University of Akron

In Partial Fulfillment

of the Requirements for the Degree

Master of Science

Mohammad Marhabaie

August, 2013 
PROTEIN COMPOSITION CORRELATES WITH THE MECHANICAL PROPERTIES OF SPIDER (ARGIOPE TRIFASCIATA) DRAGLINE SILK

\author{
Mohammad Marhabaie
}

Thesis

Approved:

Advisor

Dr. Todd A. Blackledge

Committee Member

Dr. Thomas C. Leeper

Committee Member

Dr. Richard L. Londraville

Committee Member

Dr. Matthew D. Shawkey
Accepted:

Department Chair
Dr. Monte E. Turner

Dean of the College

Dr. Chand Midha

Dean of the Graduate School

Dr. George R. Newkome 


\begin{abstract}
We investigated the natural variation in silk composition and mechanical performance within and among individual spiders of Argiope trifasciata at multiple spatial and temporal scales. Major ampullate spider silk consists of two proteins (known as MaSp1 and MaSp2) with different amino acid compositions and proposed microstructures. We assessed the reliability and precision of the Waters AccQ-Tag ${ }^{\mathrm{TM}}$ amino acid analysis kit for spider silk. Variation in chemical composition was not detectable within silk spun by a spider on a single day. However, we found that variation within a single spider's silk across days could be greater than variation among individuals. We showed that major ampullate silk of a single species of orb spiders varies in chemical composition among individuals, but is in general homogeneous among individuals of the same population. Most of the variation in silk composition in our investigation resulted from a small number of outliers (three out of sixteen individuals) with a recent history of stress. Based on reported sequences for MaSp genes, we developed a model showing the covariation of the most abundant amino acids in major ampullate silk. The model supports that the silk composition is mostly determined by relative abundance of MaSp1 and MaSp2. Finally, we showed that silk composition (proline content) strongly correlates with shrink capacity after supercontraction as well as their breaking strains. This
\end{abstract}


suggests that spiders are able to change the relative expression rate of different MaSp genes to produce silk fibers with different mechanical properties. 


\section{DEDICATION}

To my mother, Parvin, for her loving support and constant encouragement.

To my late father, Ali, for giving me confidence that I could accomplish anything that I set my sights on.

To my wife, Shiva, for her understanding when I could not spend the time with her that she deserved. 


\section{ACKNOWLEDGMENTS}

I express my gratitude to my advisor Dr. Todd Blackledge for his continual guidance, patience, and support throughout my research. I thank my committee members Dr. Thomas Leeper, Dr. Richard Londraville, and Dr. Matthew Shawkey for their input on my research and the writing of this thesis. I thank Lance Johnson and Dakota Piorkowski for helping in sample collection, Bryan Dowdell for providing assistant in sample collection and hydrolyzation, Stephanie Bilinovich and Ram Khattri for supporting the HPLC, and Samuel Evans and Kelly Le for their comments on the manuscript. I acknowledge the National Science Foundation and the Department of Biology in providing the support and research facilities used in this work. 


\section{TABLE OF CONTENTS}

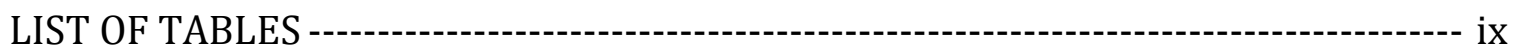

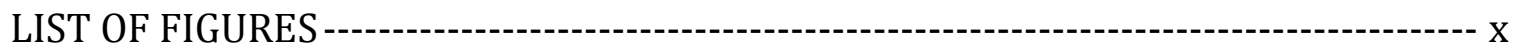

\section{CHAPTER}

I. INTRODUCTION ---:-- 1

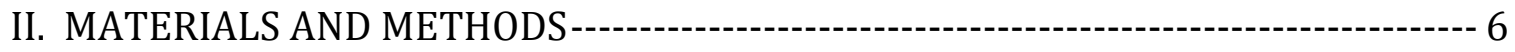

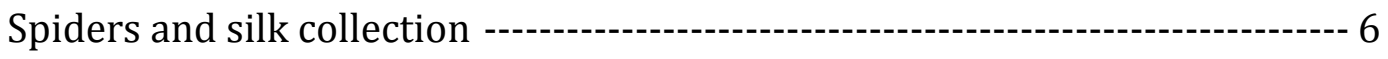

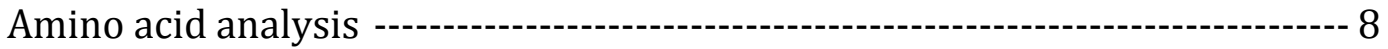

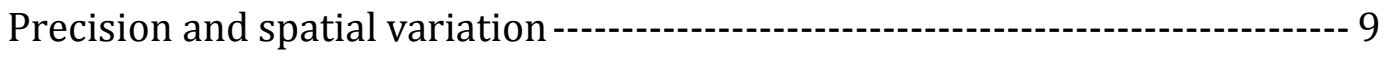

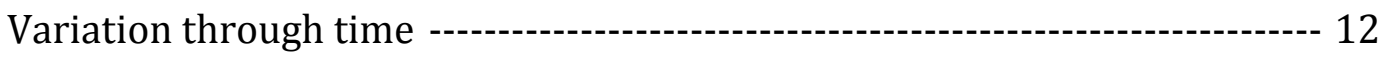

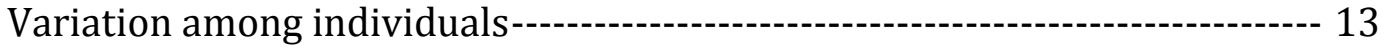

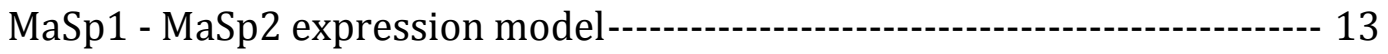

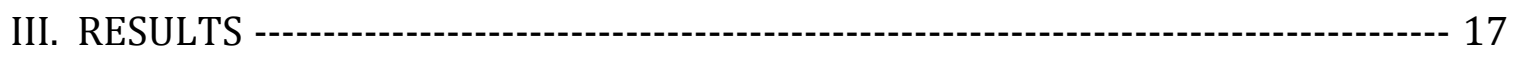

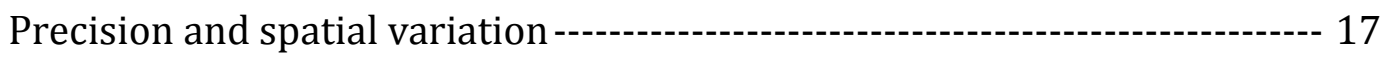

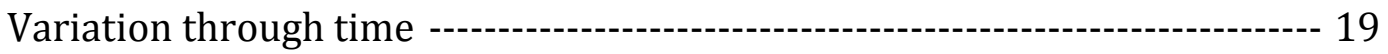

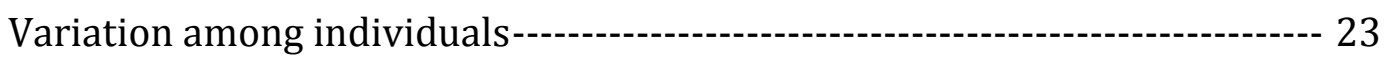




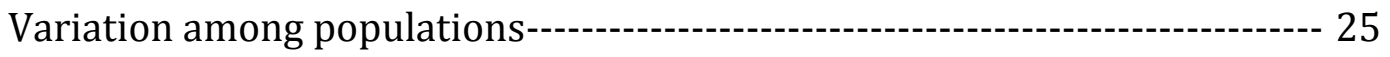

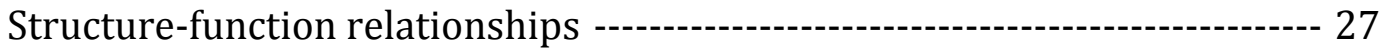

Does MaSp1/MaSp2 expression explain variation in major ampullate silk

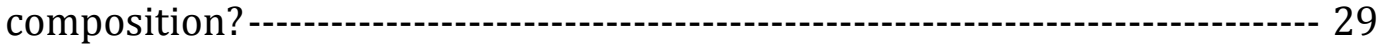

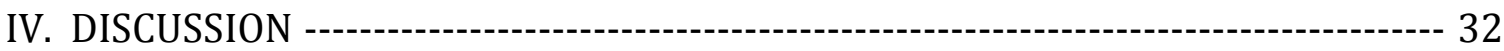

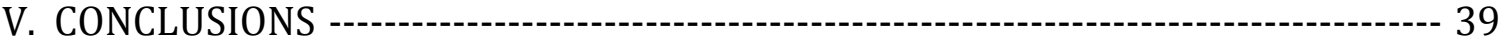

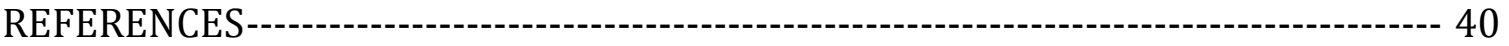




\section{LIST OF TABLES}

Table

Page

1 Estimated amino acid composition of MaSp1 and MaSp2 in Argiope trifasciata ..................................................................................................... 16 


\section{LIST OF FIGURES}

Figure

1 Sampling scheme

2 Estimated amino acid profile of dragline silk in Argiope trifasciata

3 Natural variation in proline content within $20 \mathrm{~m}$ of single draglines compared with measurement precision

$4 \quad$ Natural variation in proline content of dragline silk as a function of distance and time

5 Natural variation in proline content of dragline silk within and among days

6 Natural variation in proline content of dragline silk among individuals 24

7 Comparison of mean \pm SD amino acid compositions of dragline silk of Ohio and Florida spiders

8 Correlations between proline content and the mechanical properties of major ampullate silk

9 Correlations between proline and the three most abundant amino acids in major ampullate silk and the lack of correlation between proline and tyrosine 


\section{CHAPTER I}

\section{INTRODUCTION}

Spiders can produce up to six different types of silk fibers each specialized for a specific task (Eisoldt et al., 2011). Among these, major ampullate silk has gained more attention mostly because of its exceptionally high toughness (Agnarsson et al., 2010; Blackledge and Hayashi, 2006). This biomaterial consists of two large proteins (250-350 kDa), with different amino acid compositions known as Major Ampullate Spidroins and identified as MaSp1 and MaSp2 (Lewis, 1992). Major ampullate silk is a composite of rigid nanocrystals that provide strength and stiffness embedded in an amorphous network that provides extensibility to the fibers. cDNA analyses of major ampullate silk glands of orb web spiders (Orbiculariae) revealed that alanine and glycine are more abundant in MaSp1 while proline occurs almost exclusively in MaSp2 (Ayoub et al., 2007a). Alanine and glycine promote $\beta$-sheets that stack together to form the nanocrystals while proline disrupts the formation of $\beta$-sheet crystals and instead promotes $\beta$-spiral in the amorphous region of silk (Nelson and Cox, 2000). Hence, MaSp1, with high glycine and alanine content, is particularly important for formation of the $\beta$-sheet crystalline region of silk fibers and gives strength and stiffness to the fibers. On the 
other hand, MaSp2 with high proline content, mostly occupies the amorphous regions connecting the $\beta$-sheet crystals to each other and hence increases the extensibility of the fibers (Eisoldt et al., 2011; Liu et al., 2008a; Liu et al., 2008b; Savage and Gosline, 2008a; Hayashi et al., 1999). The combination of these two proteins with notably different compositional elements makes spider silk a good model to study structure-function relationships. It might be beneficial for orb web spiders to be able to change their silk composition for different environments. Blamires et al. (2010) and Tso et al. (2005) reported the effect of diet on the composition of major ampullate silk, however these studies only gave a rough estimate of intraspecific variation in silk composition.

Several studies highlighted supercontraction, the ability to contract after water exposure, as an important property from the structure-function standpoint (Elices et al., 2011; Boutry and Blackledge, 2010; Work, 1977). By interrupting the crystalline intermolecular bonds, water molecules change the arrangement of microstructure and allow the major ampullate silk to contract (Work, 1977). There is a significant positive correlation between proline and shrink capacity and it is hypothesized that proline, in the presence of water, gives mobility to the silk microstructure by providing more sites for water molecules to make hydrogen bonds. Therefore, proline facilitates the movement of $\beta$-sheet crystals and increases the shrink capacity (Elices et al., 2011; Boutry and Blackledge, 2010; Liu et al., 2008a; Liu et al., 2008b; Work, 1981). Even though the correlation between proline 
and shrink capacity has been shown at the interspecific level, whether this is the case at intraspecific level is not well understood.

To understand structure-function relationships we need reliable methods to measure mechanical properties and chemical composition of a fiber. In addition to the silk composition, spinning conditions (e.g. silking speed, shear forces, hemolymph $\mathrm{pH}$ and etc.) can affect the silk microstructure and ultimately its mechanical properties (Vollrath et al., 2001; Madsen et al., 1999). However, Elices et al. (2011) reported that supercontraction acts as a "reset switch" that relaxes the arrangement of silk microstructures to a defined mechanical ground state by removing most of the spinning effects helps to consistently measure the mechanical properties. Therefore, the mechanical properties of supercontracted fibers are most closely derived from their chemical compositions.

Due to difficulties in distinguishing between MaSp1 and MaSp2 with common gel electrophoresis methods, amino acid analysis has been used as an alternative method in most studies dealing with spider silk composition (Blamires et al., 2012a; Blamires et al., 2012b; Craig Vierra et al., 2011; Liu et al., 2008b; Tso et al., 2005). Proline is abundant in MaSp2 cDNA while MaSp1 is almost proline-free (Garb et al., 2010; Ayoub et al., 2007a; Garb et al., 2006). Thus, many recent studies focus on proline in major ampullate silk as an indicator of the relative content of MaSp1 and MaSp2 and hence the major determinant of the mechanical performance of a dragline silk. Most of these studies involve investigation of variation across different 
spider species (Elices et al., 2009; Liu et al., 2008b). However, spinning morphology and physiology likely varies among species in ways that may correlate with or enhance the effects of protein composition on silk properties. Our study contrasts in that it focuses exclusively on variation within a single species, so that differences in amino acid composition more accurately reflect variation in gene expression per se.

While several studies showed consistent chemical composition of major ampullate silk among individuals of the same species (Liu et al., 2008b; Casem et al., 1999; Lombardi and Kaplan, 1990), other studies suggest that amino acid composition of major ampullate silk is variable at intraspecific level (Blamires et al., 2012b; Tso et al., 2007; Tso et al., 2005; Craig et al., 2000; Work and Young, 1987). Most of these studies used mixtures of several meters of major ampullate fiber to get the required quantity of silk for amino acid analysis, and none of them assessed the potential variation in silk composition over a short length of a silk fiber. Many of them also did not optimize their analysis for spider silk fiber and do not assess the precision of their method. Some of them used old techniques and instruments which can decrease the precision of the method. Here, by using the Waters AccQ-Tag ${ }^{\mathrm{TM}}$ amino acid analysis kit, we propose a reliable and more sensitive method to analyze the composition of major ampullate silk with very low quantity of material (as low as $150 \mathrm{ng}$ or $3 \mathrm{~cm}$ of silk). More importantly, we assessed the reliability and precision of amino acid composition analysis for spider silk. We then investigated variation in the chemical composition of major ampullate silk within and among individual 
spiders of the same species at multiple spatial and temporal scales. Finally, we have related variation in chemical composition of silk to mechanical performance. 


\section{CHAPTER II}

\section{MATERIALS AND METHODS}

Spiders and silk collection

We collected nine adult female Argiope trifasciata from the University of Akron Field Station at the Bath Nature Preserve (Bath, $\mathrm{OH}$ ) and purchased seven more adult female $A$. trifasciata from a distributor (tarantulaspiders.com) in Florida. We kept all spiders at the University of Akron's greenhouse and fed all of them with house crickets, Acheta domestica. We forcibly silked the spiders to collect pure samples of major ampullate silk under controlled conditions. To silk a spider, we anesthetized it with $\mathrm{CO} 2$ for 30-50 seconds and fixed it on a glass platform using Scotch(C) tape. We allowed the spider to recover for 3-5 minutes and then, under a stereomicroscope, carefully collected major ampullate silk. We collected samples only from a single major ampullate gland (either the right or left) for each individual.

For mechanical testing, we collected 46 samples from six individual spiders. Each spider was sampled for up to four days within a fifteen day period. We mounted the silk samples across $15.7 \mathrm{~mm}$ diameter holes in cardboard using 
cyanoacrylate adhesive (Superglue $®$ ). We estimated the diameter of each sample based on the average of three measurements taken along its length using polarized light microscopy at 1000X (Blackledge et al., 2005). We then measured the material properties (true breaking stress, true breaking strain, Young's modulus, toughness, and shrink capacity) of the samples using a Nano Bionix UTM tensile tester (Agilent Technologies, Phoenix, AZ, USA) as described previously (Boutry and Blackledge, 2010; Agnarsson et al., 2009). We normalized the mechanical properties of each sample based on its diameter. The Nano Bionix tensile tester was equipped with a humidity chamber and had a load resolution of $1 \mu \mathrm{N}$ and an extension resolution of $1 \mu \mathrm{m}$. Briefly, we mounted a sample at room temperature and humidity. We pulled the silk until it generated $20 \mathrm{uN}$ load to assure the sample was taut. We then increased the humidity to more than $75 \% \mathrm{Rh}$ within 60 seconds to supercontract the sample. After maximum contraction, we relaxed the sample to have a completely slacked silk and dried it to less than 5\% Rh within 90 seconds. Then, we performed a tensile test on the dried silk at a strain rate of $0.1 \mathrm{~s}-1$. We measured the shrink capacity (in percentage) as the difference in the length of the supercontracted silk and its original length divided by the original length.

For amino acid composition analysis, we collected $4 \times 105 \mu \mathrm{m} 3(9-15 \mathrm{~cm}$ in length) samples of major ampullate silk. First, we measured the diameter of major ampullate silk. Then based on the diameter and the required volume, we calculated and collected the corresponding length $(9-15 \mathrm{~cm})$ of the dragline. As the dragline came out of the major ampullate silk gland, we wrapped it around a clean thin glass 
rod (0.2-0.3mm in diameter) attached to a rotating axel. The silk was wrapped around the terminal end $(1-2 \mathrm{~cm})$ of the rods. We then cut the terminal end and transferred it to the bottom of a $6 \times 50 \mathrm{~mm}$ glass test tube. We used powder free gloves during the entire procedure to minimize contamination.

Amino acid analysis

We used the established protocol described by Smith (2003) to perform vapor phase hydrolyzation and amino acid analysis. We used a hydrolysis vial with a PTFE cap (Eldex Laboratories, Napa, CA, USA), which holds fourteen $6 \times 50 \mathrm{~mm}$ glass test tubes, each containing an individual silk sample. However, to minimize the possibility of cross contamination, we placed up to eight prepared test tubes containing silk into the hydrolysis vial and filled the vacant spaces with blank tubes. We used the blank samples to estimate the cross contamination among samples in a single hydrolysis vial. We added $400 \mathrm{ul}$ of $6 \mathrm{~N} \mathrm{HCl}$ to the bottom of the hydrolysis vial and vacuum-sealed it using a vacuum pump with an ultimate vacuum of $5 \mathrm{~Pa}$. We hydrolyzed samples under $6 \mathrm{~N} \mathrm{HCl}$ vapor at $115^{\circ} \mathrm{C}$ for 21 hours. Afterwards, we removed the inner sample tubes, dried them under vacuum, and derivatized them with an aminoquinolyl-NHS compound (AQC) using a Waters AccQ-Tag ${ }^{\mathrm{TM}}$ kit (Waters, Milford, MA, USA). We separated the derivatized amino-acids with a $3.9 \times 150 \mathrm{~mm}$ C18 column from the AccQ-Tag ${ }^{\mathrm{TM}}$ kit using an ÄKTAPurifier highperformance liquid chromatography (HPLC) system with UV detection. We analyzed 
chromatographs and integrated peak areas using Unicorn 5.20 software (GE

Healthcare, Pittsburgh, PA, USA). We calibrated the instrument with five amino acid standard concentrations (50-1000pmol) and used the calibration file to calculate the relative content of each amino acid as mole percent per 100 residues. Because asparagine and glutamine are deaminated to their respective acids under $\mathrm{HCl}$ hydrolyzation, we reported them as mixtures of asparagine/aspartic acid and glutamine/glutamic acid. Moreover, methionine and cysteine are recovered in low non-quantifiable yield, tryptophan will be destroyed, and cystine will be recovered as cysteine. We performed HPLC at room temperature, thus, we were not able to distinguish between arginine and threonine peaks and we reported them as a mixture of arginine/threonine. Much of our analysis focuses on the percentage of proline in silk because this amino acid occurs almost exclusively in MaSp2 (Blamires et al., 2012b; Liu et al., 2008b; Gatesy et al., 2001).

Precision and spatial variation

Little is known about the potential for variation in amino acid composition of spider silk over small spatial scales (e.g. within $\mathrm{cm}$ along the same fiber). Most prior work either used longer lengths of threads, thereby averaging out any variation, or too small a sample size to detect variation in composition. Moreover, the precision with which the amino acid composition of silk can be determined has never been quantified. Thus, it is impossible to know the degree to which minor variation in 
measurements of amino acid compositions among silk fibers might be biologically meaningful versus simple error. To check the reliability of our amino acid analysis method, and whether or not major ampullate silk composition varied across a single dragline, we silked three spiders (identified as \#25, 30 and 37 hereafter) on three separate days within a seven day period. On each sampling day, we collected three single-position samples (S1, S2 and S3 in Figure 1) at 10 meter intervals along with three additional sub-samples from each of those locations (Figure 1). One subsample from each location would later be combined into a single blended sample according to their relative position along the silk fiber to make a total of three blended samples (A, B and C in Figure 1). These blended samples presumably averaged any real chemical variation across the silk fiber so that they should have identical compositions and therefore provide a strong estimate of the error inherent in the amino acid analysis itself. The length of each individual sub-sample was one third of the length of each single-position sample. Thus, the volumes of all final six samples were equal. At the end of the third location, we collected 5-10 cardboardmounted samples (region M in Figure 1) for mechanical testing so that their chemical composition could be inferred from the last single-position sample (S3 in Figure 1). We calculated the precision of our method by taking the average of the coefficients of variation of the blended samples for the major amino acids in spider dragline (Glycine, Alanine, Proline, Serine and Glutamine/Glutamic acid). These amino acids comprised more than $90 \%$ of a dragline silk, thus they better represent method precision compared to less abundant amino acids. To examine whether or 
not the variation in silk composition within 20 meters of a single major ampullate silk fiber was significantly different from the measurement precision, we ran F-tests comparing the variances of the three single-position samples versus the three blended samples for each individual spider (five F-tests in total). Then, we calculated the overall p-value using a Fisher's combined probability test.

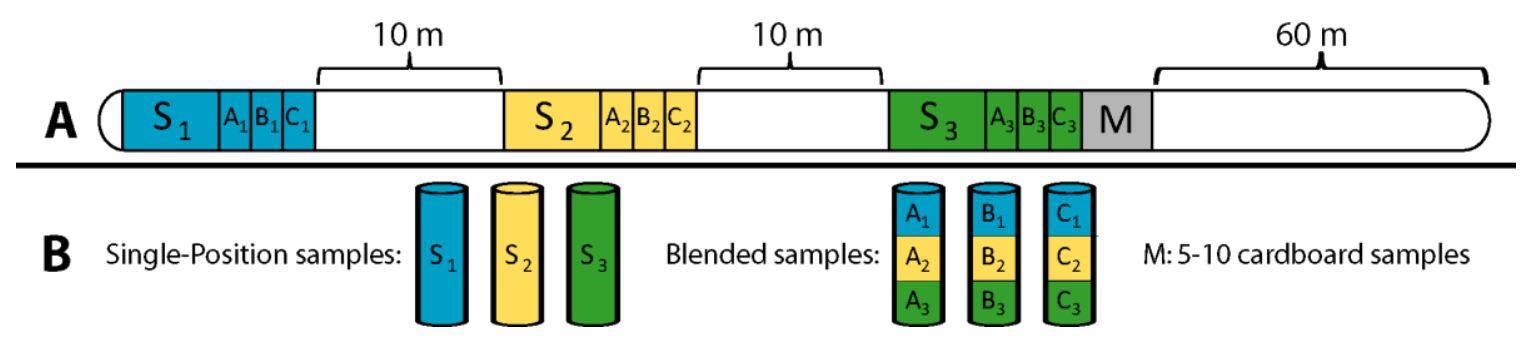

Figure 1: Sampling scheme. Panel A shows the relative position of the samples on a silk fiber. Panel B shows the final six samples for amino acid analysis. Variation within single draglines was assessed by taking samples at the beginning, middle, and end of 20m of a single dragline (S1, S2 and S3). Measurement precision was assessed by combining silk from each of those three locations into three blended cocktails (A, B and C), each of which was independently hydrolyzed and analyzed. The blending should have assured homogeneous composition among the samples. We collected 5-10 cardboard-mounted samples from the M region for mechanical testing and then continued the silking process for an additional $60 \mathrm{~m}$ to ensure that the next sampling day's collections were at a significantly greater spatial distance than any comparisons within a single day. 
Variation through time

To compare variation in silk composition among days, we used the data from all the above spiders (\#25, 30 and 37) and two more individual spiders (\#22 and 62) for three consecutive days and one more spider (\#52) for three separate days over a ten day period. In addition, we silked spider 25 for one more day (the fourth sampling day), which was a total of fifteen calendar days after the spider was first sampled. On each day, we collected two samples for amino acid analysis and 5-10 cardboard-mounted samples for mechanical testing. We took care to minimize the space between samples, so the chemical composition of cardboard samples could be inferred from the amino acid samples. Finally, we continued the silking process for 60 meters at the end of each day. Spiders have a reservoir of liquid silk dope of unknown volume waiting to be spun into silk. We had no way to assess how much silk any spider might have spun outside of our sampling but this protocol did ensure that the next sampling day's collections were at a significantly greater spatial distance than any comparisons within a single day. Because of the poor understanding of how the silk dope may or may not be regulated physiologically by the spider, we also considered how silk fibers might vary both as a function of the distance from prior samples (a measure of material removed from the reservoir) and as time since last collection (presumably a measure of potential for physiological manipulation of the reservoir). 
Variation among individuals

To compare variation in chemical composition among individual spiders, we used the data from all six spiders above and collected 1-4 amino acid samples from 10 more $A$. trifasciata. Six of those spiders were from a different population (designated here as Florida spiders). We purchased them from a distributor and shipped them to the laboratory. Therefore they were not only a geographically distinct population, but they also likely were under greater physiological stress prior to the experiment, compared to "Ohio" spiders.

MaSp1 - MaSp2 expression model

Several studies suggest that variation in major ampullate silk composition is potentially determined by the relative abundance of MaSp1 and MaSp2 (Blackledge, 2012; Boutry and Blackledge, 2010; Tso et al., 2005). However, other factors can affect the silk composition, such as expression of other silk genes in the major ampullate gland (Garb et al., 2006), potential post-translational modifications (currently uninvestigated), and the existence of multiple alleles for some MaSp proteins (Ayoub and Hayashi, 2008).

If the composition of major ampullate silk is mostly determined by the relative abundance of MaSp1 versus MaSp2 proteins, in theory, the amino acid composition of silk should vary proportionally across a defined set of values 
determined by the MaSp1/MaSp2 ratio. At one end of this set, there is an amino acid profile of a pure MaSp1 silk - which is exactly the same as the profile of a single MaSp1 molecule - and at the other end there is a profile of a pure MaSp2 silk (Figure 2). Thus, by knowing the ratio of MaSp1/MaSp2 we can predict the whole amino acid profile of a silk fiber. Furthermore, proline is an indicator of MaSp2. Therefore, knowing the proline ratio should be enough to predict the silk amino acid profile.

With this assumption and based on reported sequences for the repetitive regions of MaSp1 (14 repetitive units in total 549bp) and MaSp2 (18 repetitive units in total 851bp) in A. trifasciata (accession \# AAZ15371, AAK30596 and AAK30595), we estimated the amino acid composition of a pure MaSp1 and a pure MaSp2 dragline silk (Table 1) to evaluate the expected linear changes between different pairs of amino acids as expression level changes (Figure 2). We then used the slope of the estimated model and calculated the intercept of the line as the average of data points for each axis. We plotted our real data and compared them to the best fit of the model to assess how well a simple model of variation in MaSp1/MaSp2 expression explained the variation in our dataset, compared to alternative explanations such as expression of other silk genes in the major ampullate gland, contamination, or experimental error. 


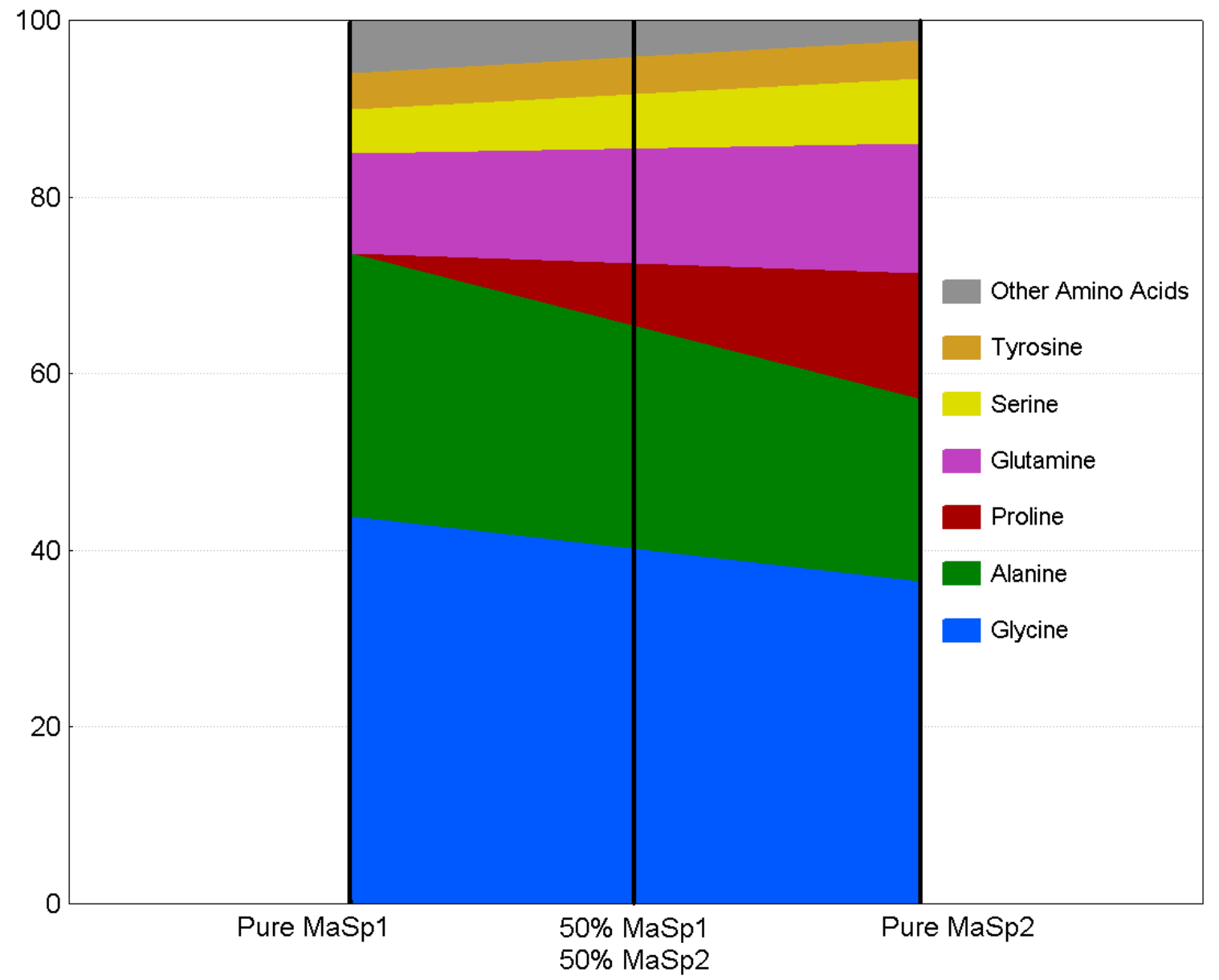

Figure 2: Estimated amino acid profile of dragline silk in Argiope trifasciata. Amino acid composition of dragline silk varies proportionally across a defined set of values determined by the MaSp1/MaSp2 ratio. The amino acid composition of MaSp proteins are estimated based on the repetitive regions of reported sequences for MaSp proteins in Argiope trifasciata. 
Table 1: Estimated amino acid composition of MaSp1 and MaSp2 in Argiope trifasciata. There is no proline in the repetitive regions of MaSp1 cDNA.

\begin{tabular}{|lcc|}
\hline Amino Acid & MaSp1 & MaSp2 \\
\hline Glycine & 43.9 & 36.55 \\
Alanine & 29.69 & 20.56 \\
Proline & - & 14.22 \\
Serine & 5.1 & 7.4 \\
Glutamine & 11.29 & 14.69 \\
Tyrosine & 4.01 & 4.35 \\
Arginine & 0.91 & 1.06 \\
Threonine & 0.36 & 0.47 \\
Valine & 0.55 & 0.59 \\
Leucine & 3.1 & - \\
Aspartic acid & 0.36 & 0.12 \\
Phenylalanine & 0.18 & - \\
Glutamic acid & 0.55 & - \\
\hline
\end{tabular}




\section{CHAPTER III}

\section{RESULTS}

Precision and spatial variation

We calculated the precision of our method by taking the average of the coefficients of variation calculated from the three blended samples (coefficient of variation: Glycine $=0.012$, Alanine $=0.013$, Proline $=0.014$, Serine $=0.019$ and Glutamine/Glutamic acid=0.026). The total amino acid content of each sample was between $0.5-6 \mathrm{nmol}$ and the cross contamination among samples in a single hydrolysis vial was less than $1 \%$ of the total amino acid content of each sample. Thus, cross contamination did not affect the results significantly. Figure 3 compares the proline content of single-position and blended samples in three spiders collected over multiple days. Although two outlier data points (spider 37-day 3 and spider 30day 1) suggest variation in chemical composition within 20 meters of major ampullate silk, the coefficients of variation of single-position and blended samples were not statistically different $(p=0.23$, Figure 3$)$. Moreover, the two outlier data points were partially hydrolyzed samples. We realized this by checking the alanine and alanine/glycine values which were lower than the acceptable range for major 
ampullate silk in $A$. trifasciata (Table 1 shows the acceptable ranges). Therefore, any potential variation in chemical composition within 20 meters of major ampullate silk fiber was below the detection level of our method.

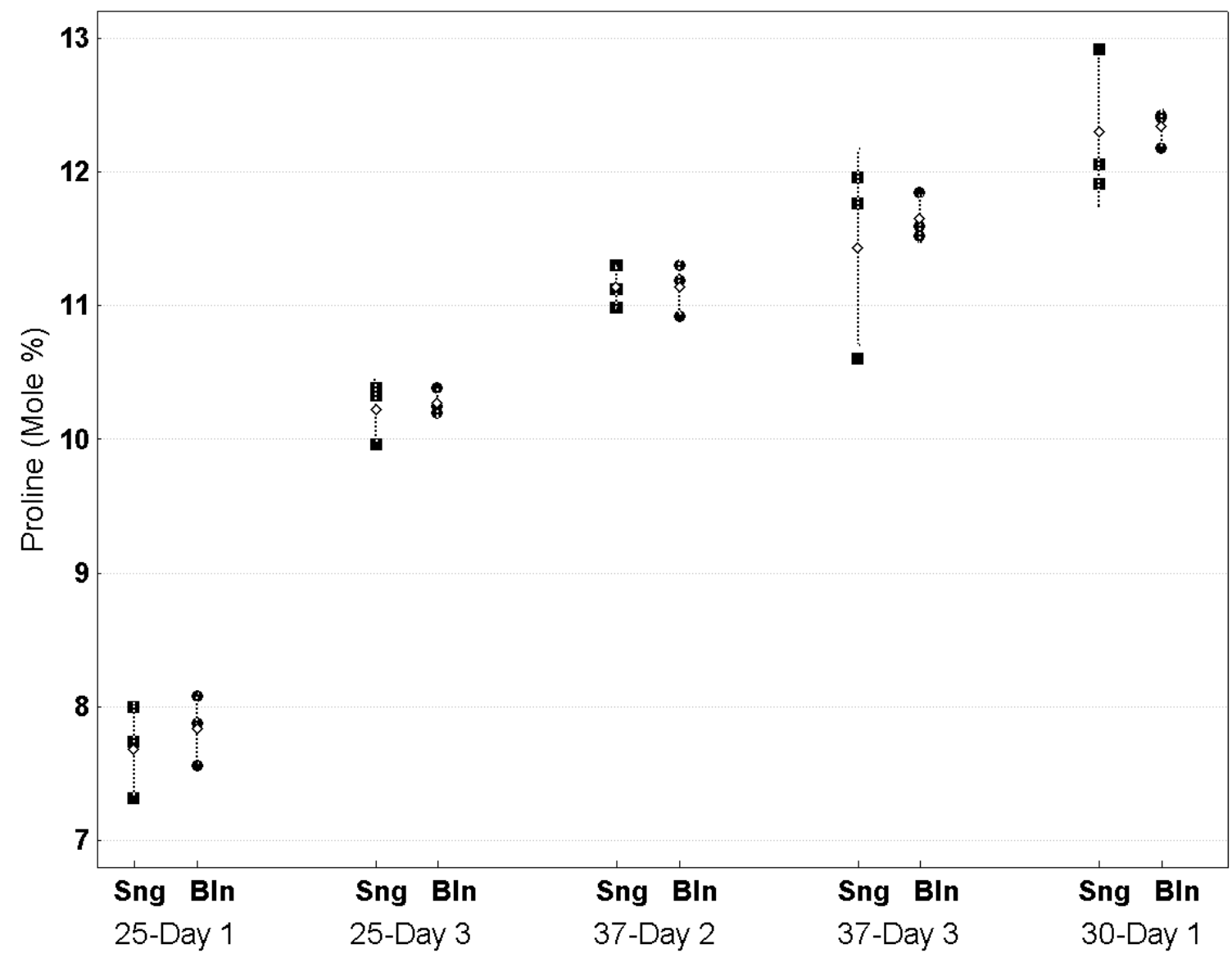

Figure 3: Natural variation in proline content within $20 \mathrm{~m}$ of single draglines compared with measurement precision. The open diamonds correspond to the mean proline content of the dragline and the dashed lines show standard deviation. Fisher's combined probability test showed that variation in proline content (cont.) 
within $20 \mathrm{~m}$ of single draglines was no greater than measurement precision $(p=0.23)$. Single and blended samples are described in Figure 1. The spider identities and day numbers correspond to other figures. Sng = single-position sample, Bln = blended sample.

Variation through time

We found that silk proline content could vary in an individual over multiple days. Proline increased by $80 \%$ (from $7.3 \%$ to $13.1 \%$ of all amino acids) after fifteen days in spider 25 and decreased by $62 \%$ (from $8.5 \%$ to $5.3 \%$ ) after three consecutive days in spider 62 (Figure 4). However, silk proline content was constant over 20 meters of silk collected continuously in a single day for most spiders and 100 meters for spider 56 (Figure 5). Silk composition was remarkably homogeneous within most individuals and typically varied by less than $\pm 2 \%$ across three days (Figure 4). The average coefficient of variation within a single day (0.02) was significantly lower than the average coefficient of variation over multiple days $(0.08$, Figure 5). 

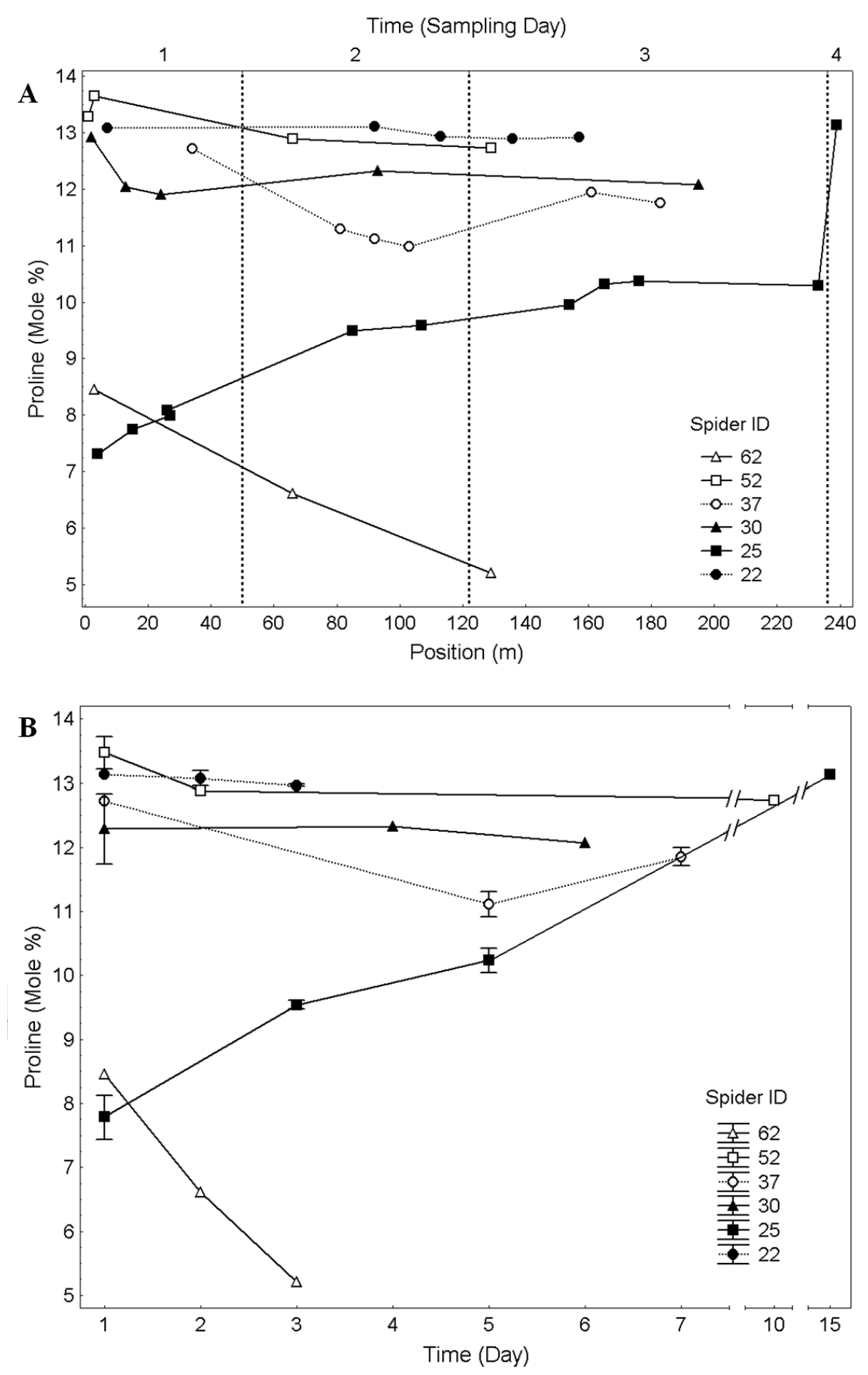

Figure 4: Natural variation in proline content of dragline silk as a function of distance and time. Panel A shows the variation in proline content of the silk (cont.) 
collected on different collection days (not necessarily contiguous) from six spiders against the position of the samples along the reeled silk. Position is the relative distance of the sample to the total length of the reeled silk. Spiders might produce and use more silk than we collected. Proline variation was minimal both within draglines and across spiders, with the exception of two individuals that were noteworthy for the unusually low proline content of their silk. Panel B shows the same data expressed as day of collection instead of distance of dragline reeled from the spider. The spider identities correspond to other figures. 


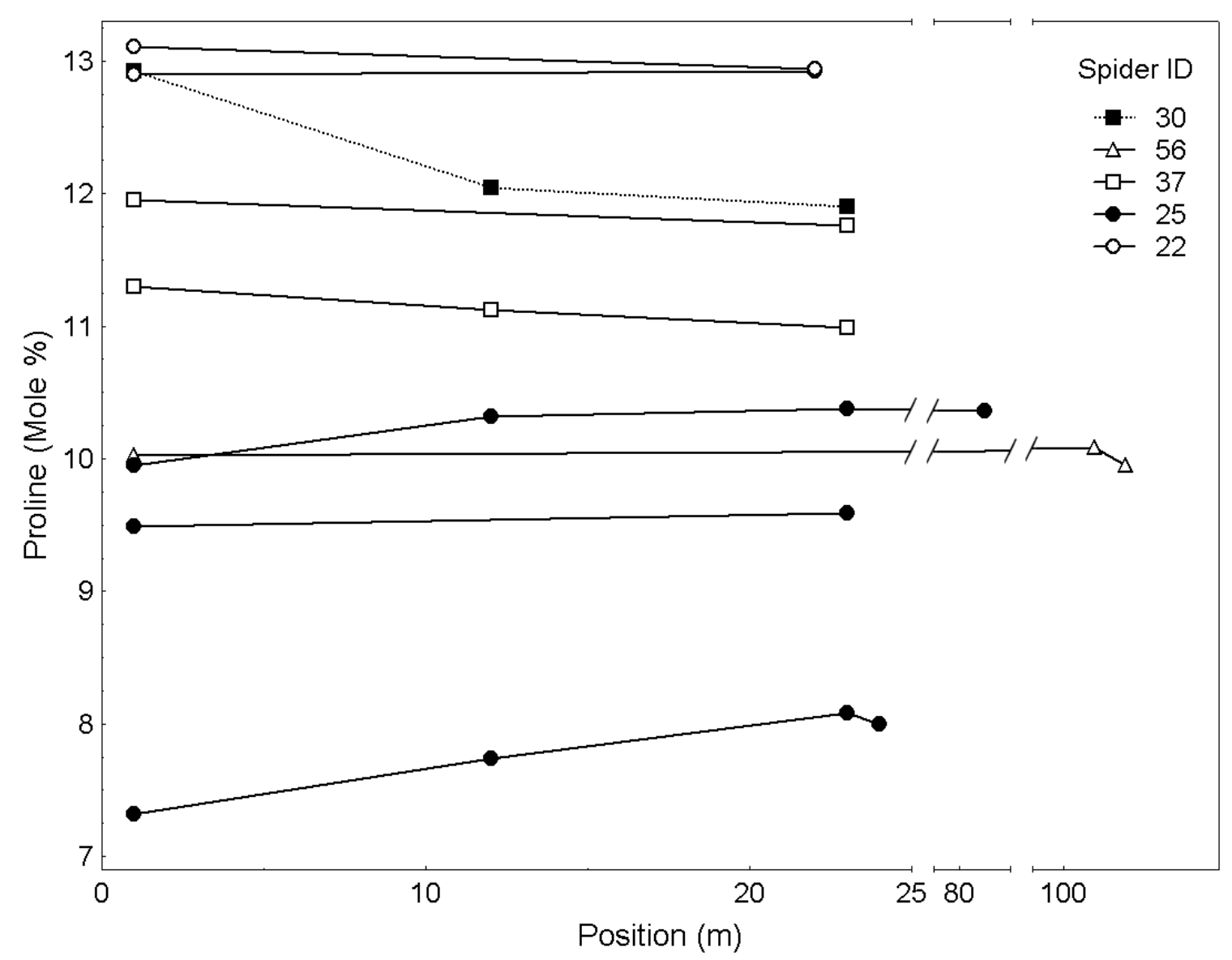

Figure 5: Natural variation in proline content of dragline silk within and among days. Each line connects samples collected in the same day. Five spiders are compared, most for multiple days. The spider identities correspond to other figures. Silk proline content is consistent within a single day, while it varies among days. 
Variation among individuals

The proline content of major ampullate silk varied from $5.2 \%-13.6 \%$ among individuals $A$. trifasciata. Despite this broad range, proline content was remarkably homogeneous among most individuals, varying by less than $\pm 2 \%$ among thirteen of sixteen individuals (Figure 6). Moreover, silk proline content was more consistent with in populations, varying by less than $\pm 1.5 \%$ among most individuals of the same population. We showed that the variation in chemical composition is not detectable within silk spun on a single day. However, the variation within a single spiders' silk across days can be larger than variation among individuals. Most of the variation in silk composition in our investigation results from a small number of outliers, sampled from spiders with a recent history of stress. 


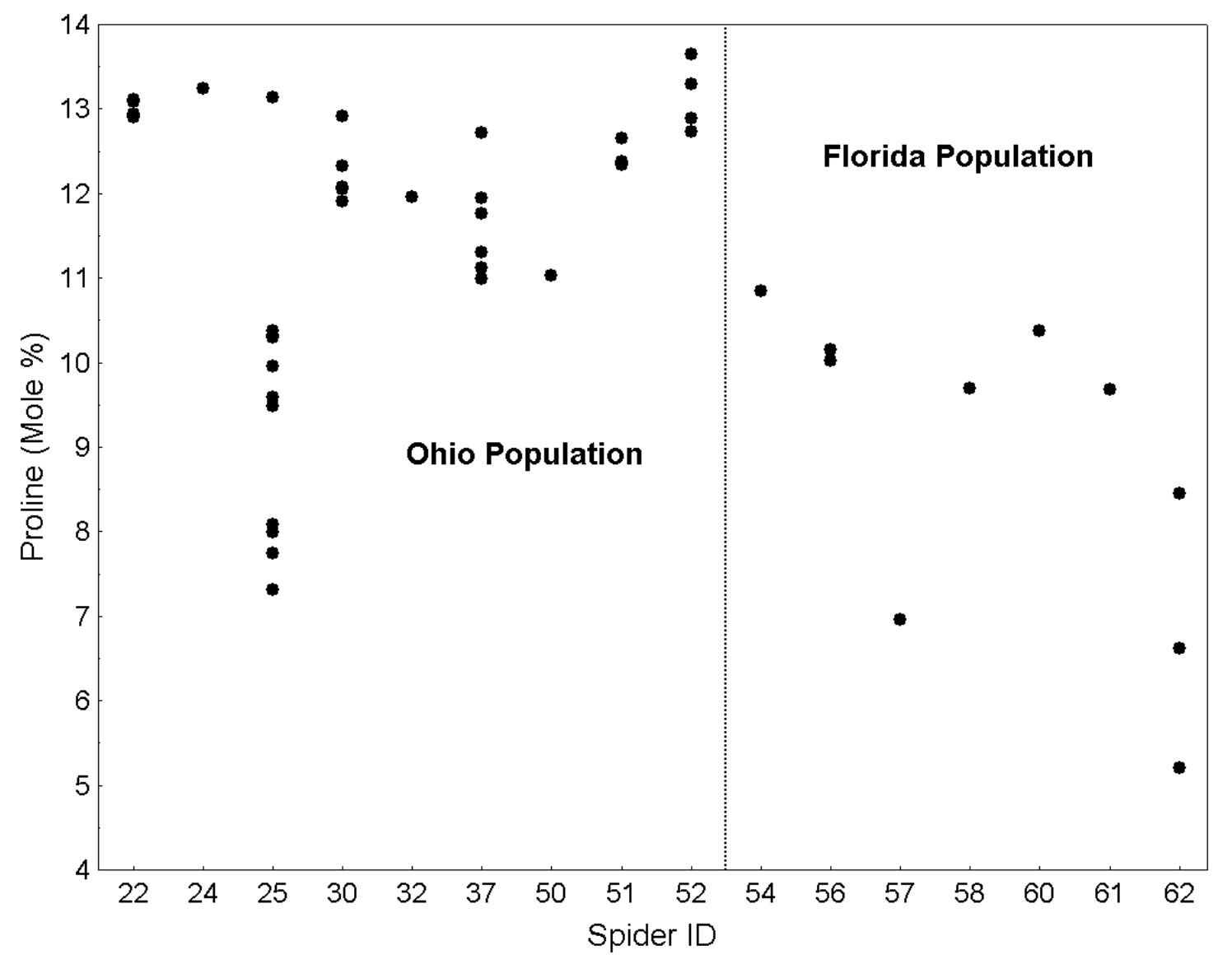

Figure 6: Natural variation in proline content of dragline silk among individuals. Sixteen spiders from two populations were compared. There is wide range of silk proline content (5.2\% - 13.6\%) among individuals of Argiope trifasciata. However, most of the variation in silk composition results from samples collected from three spider individuals with recent history of stress. Moreover, silk proline content was consistent among most individuals of the same population. Each dot represents a sample of dragline. The spider identities correspond to other figures. 
Variation among populations

The shipped spiders (Florida population) on average produced major ampullate silk with significantly lower proline content $(\mathrm{p}<0.001$, Figure 6). Overall, the amino acid compositions of major ampullate silk were significantly different between the Ohio and Florida spiders $(\mathrm{p}<0.001)$. Major ampullate silk from Florida spiders had more glycine, alanine, arginine/threonine, and phenylalanine, but less serine, glutamine/glutamic acid, and proline (Figure 7). These correlations are consistent with the MaSp1-MaSp2 expression model (Figure 9) and suggest that Ohio spiders express more MaSp2 in their major ampullate silk. We emphasize that this variation correlates with major differences in how the two groups of spiders were handled, in addition to their geographic origins, as the Florida spiders were shipped through the mail enduring a period of protracted stress. 

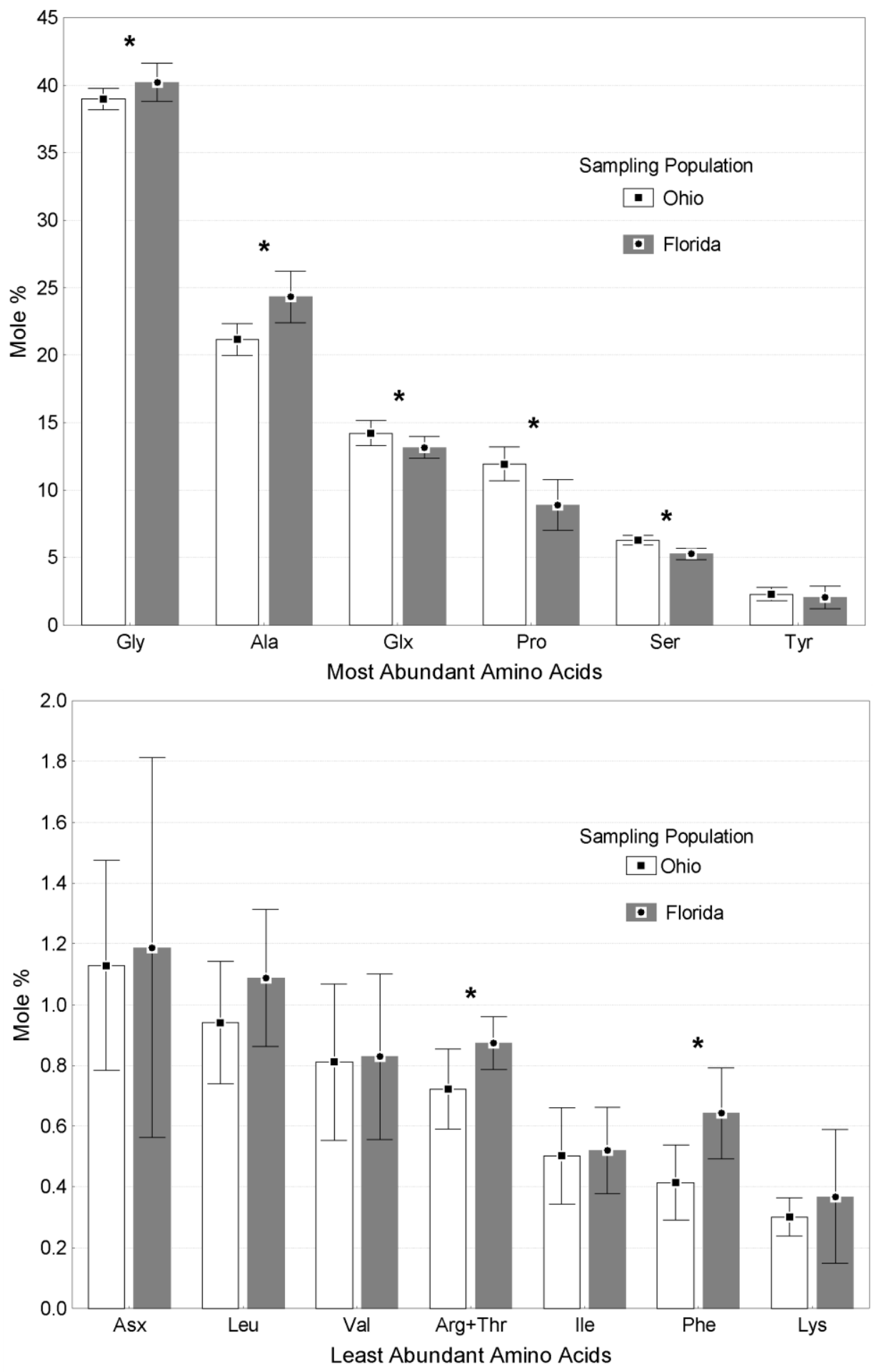

Figure 7: Comparison of mean \pm SD amino acid compositions of dragline silk of Ohio and Florida spiders. We assessed 34 samples from nine individuals of Ohio (cont.) 
spiders and 11 samples from seven individuals of Florida spiders. Asterisks show statistically significant pairwise comparisons $(\mathrm{p}<0.05)$. Three letter amino acid abbreviations are used. Because asparagine and glutamine will be deaminated to their respective acids under $\mathrm{HCl}$ hydrolyzation, we report them as mixtures of asparagine/aspartic acid (Asx) and glutamine/glutamic acid (Glx). Cystine and methionine are non-quantifiable in $\mathrm{HCl}$ hydrolyzation process, thus, they are excluded from this analysis. Amino acids associated with MaSp2 (Glx, Pro, and Ser) are higher in Ohio spiders and amino acids associated with MaSp1 (Gly and Ala) are higher in Florida spiders. There is no difference in tyrosine which is expected, because tyrosine is nearly equally abundant in both MaSp1 and MaSp2 cDNA.

Structure-function relationships

Amino acid composition correlated strongly with some aspects of the mechanical performance of the silk (Figure 8). In particular, we found a strong correlation between major ampullate silk proline content and its shrink capacity after supercontraction $(\mathrm{R} 2=0.75, \mathrm{p}<0.001)$. The correlation is positive and plateaus after reaching $13 \%$ proline. Proline content also strongly correlated with true breaking strain $(R 2=0.75, p<0.001)$, but not with true breaking stress $(R 2=0.13$, $\mathrm{p}=0.32$ ). There was a weak correlation between proline content and toughness $(\mathrm{R} 2=0.24, \mathrm{p}=0.09)$ whereas Young's modulus did not correlate with silk proline content $(\mathrm{R} 2=0.17, \mathrm{p}=0.20)$. 

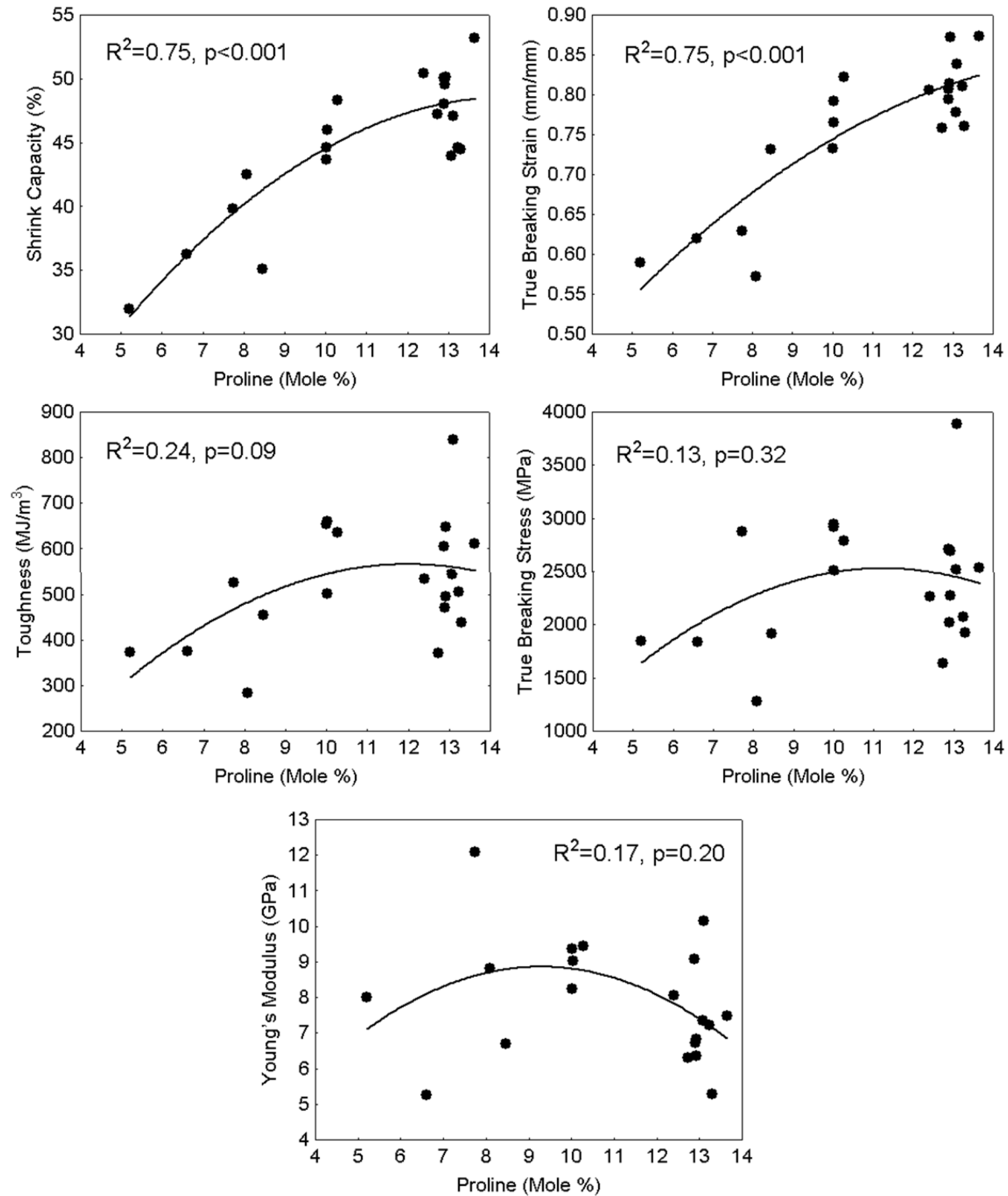

Figure 8: Correlations between proline content and mechanical properties of major ampullate silk. Each point is the average of as many as four mechanical test replicates within $50 \mathrm{~cm}$ of a single sample for amino acid analysis. Data are (cont.) 
from multiple locations along draglines from six spider individuals. In total, 46 mechanical tests and 20 amino acid samples were examined. Polynomial (quadratic) regression is used to obtain the best fit. There is a strong correlation between major ampullate silk proline content and its shrink capacity after supercontraction. Proline is also strongly correlated to strain and weakly correlated to toughness. Neither stress nor modulus correlates with proline content.

Does MaSp1/MaSp2 expression explain variation in major ampullate silk composition?

We compared our amino acid data (45 samples from 16 spider individuals) to the described MaSp1-MaSp2 expression model to assess how well MaSp1 versus MaSp2 expression explains the variation in silk composition (Figure 9). The model explained the majority of the variation in alanine $(75 \%)$ and much of glutamine/glutamic acid (51\%). Due to the three outlier samples in the glycine graph (open circles in Figure 9), the model only explained $44 \%$ of the variation in glycine relative to proline. By removing the three outlier samples in the glycine graph from all analyses (open circles in Figure 9), the model explained most of the variation in alanine (83\%), glycine (83\%), and half of glutamine/glutamic acid (50\%) relative to proline. In addition, there was no meaningful correlation between tyrosine and proline (Figure 9), which was predicted by the model because tyrosine is nearly equally abundant in MaSp1 and MaSp2 cDNA (4.01\% and 4.35\% 
respectively). The absolute values measured for tyrosine were less than expected because tyrosine is recovered in low yield under $\mathrm{HCl}$ hydrolyzation.
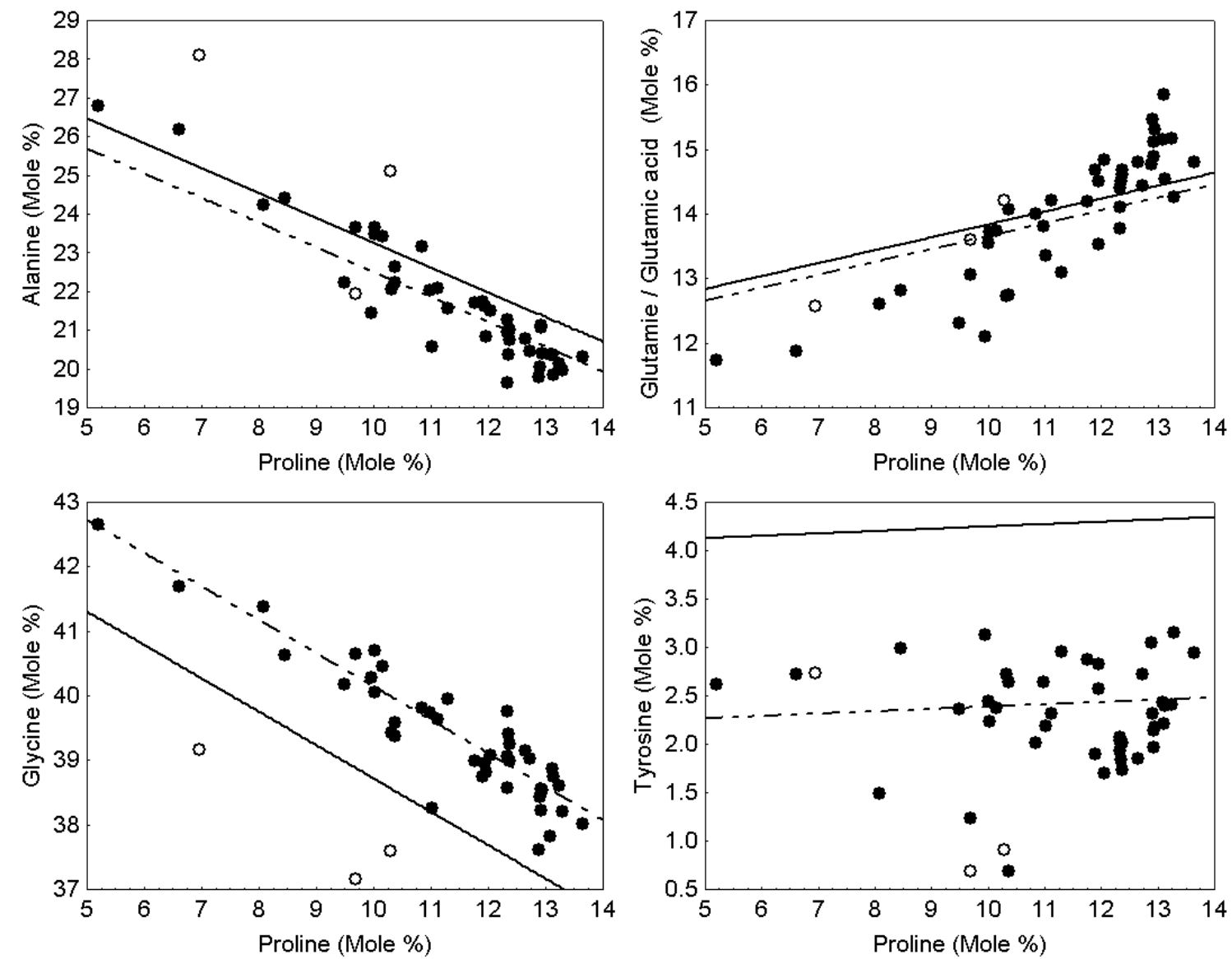

Figure 9: The correlation between proline and the three most abundant amino acids in major ampullate silk and the lack of correlation between proline and tyrosine. In each plot, the sold line shows the estimated correlation which is calculated based on reported sequences for the repetitive regions of MaSp1 and MaSp2 in Argiope trifasciata, and the dashed line shows the best fit with the slope of the (cont.) 
estimated model. The open circles show the same three outlier samples in every graph. The estimated model explains most of the variation in alanine, glycine, and much of glutamine/glutamic acid. There is no meaningful correlation between tyrosine and proline which is predicted by the model as well. As tyrosine is recovered in low yield under $\mathrm{HCl}$ hydrolyzation, the values are half of the expected. 


\section{CHAPTER IV}

\section{DISCUSSION}

Our study showed that the chemical composition of major ampullate silk was homogeneous over 20 meters of a single silk fiber (100 meters in spider 56, Figure 5) and generally consistent across longer lengths of fibers produced on multiple days (Figure 4). Amino acid composition was similar within individual spiders $(0.02$ coefficient of variation) with the exception of two individuals who silk was both more variable and generally substantially lower in proline. We found consistent variation in silk composition among individuals of two populations ("Ohio" versus "Florida"), although geography was confounded with differences in how the spiders were handled. Finally, we demonstrated that variation in the chemical composition of major ampullate silk correlates with mechanical properties. In particular, prolinerich silk was more extensible and shrank more after supercontraction, further validating the model of MaSp1/MaSp2 "function" previously supported at an interspecific level (Elices et al., 2009; Liu et al., 2008a; Liu et al., 2008b; Savage and Gosline, 2008a, b). 
Hydrolyzation is a key step in amino acid analysis. The goal of this step is to break down all peptide bonds without losing free amino acids, but this is imperfect for some proteins. The classical $\mathrm{HCl}$ hydrolyzation is conducted under a vacuum at $110^{\circ} \mathrm{C}$ for 18-24 hours (Stein and Moore, 1950). However, the stability of peptide bonds between amino acids varies. The peptide bonds between hydrophobic amino acids (e.g. alanine, valine, leucine and isoleucine) are relatively stable under $\mathrm{HCl}$ hydrolyzation. If the target protein is rich in these hydrophobic amino acids, a partial hydrolyzation will lead to a significant underestimation of the hydrophobic amino acids and overestimation of the other amino acids. Since poly-alanine is a common domain in major ampullate silk, the alanine recovery is a very good indicator of the quality of hydrolyzation. When the alanine content of a sample is radically lower than the expected value, the sample should be considered cautiously.

Based on the general guideline for $\mathrm{HCl}$ hydrolyzation (Smith, 2003), we hydrolyzed our samples at $115^{\circ} \mathrm{C}$ for 21 hours and we found few partially hydrolyzed silk samples (3 out of 68). Although the recovery of free amino acids may decrease by increasing the hydrolyzation time, the relative abundance of most amino acids does not change significantly while alanine recovery increases dramatically (unpublished data). It should be noted that under $\mathrm{HCl}$ hydrolyzation tryptophan will be destroyed, cystine will be recovered as cysteine, and methionine and cysteine are recovered in low, non-quantifiable yields. However, these amino acids comprise less than $0.5 \%$ of the total amino acid composition of major 
ampullate silk (Ayoub et al., 2007b; Casem et al., 1999; Lombardi and Kaplan, 1990; Work and Young, 1987).

The complete sequences of MaSp1 and MaSp2 of Argiope trifasciata are still unknown. Therefore, to assess how well the variation in MaSp1/MaSp2 expression explains the variation in our dataset, we developed a model based on the repetitive regions of reported sequences. We used the repetitive regions because they comprise the bulk of the protein (more than 90\% in Latrodectus hesperus). Despite using incomplete sequences, the estimated model explained the majority of the variation in alanine, glycine, and much of glutamine/glutamic acid. The pattern of covariation among amino acids supports that major ampullate silk composition in $A$. trifasciata is mostly determined by the relative abundance of MaSp1 and MaSp2. The model demonstrates that the variation in proline correlates with variation in other major amino acids and suggests that proline is an indicator of the whole amino acid profile of major ampullate silk, which is why much of our analysis focuses on the percentage of proline.

While amino acid composition of major ampullate silk was consistent among days in three spiders $(22,30$ and 52$)$, it significantly $(\mathrm{p}<0.001)$ varied over multiple days in the other spiders (25, 37 and 62; Figure 4). However, the dragline silk of spiders 62 and 25 had the lowest proline level reported in A. trifasciata. Their silk behaved differently than the others and spider 25 showed a trend toward recovery to the normal state in both proline level and mechanical performance of the silk. 
Therefore, we suspect that due to the stresses that they may have encountered during the collecting, storing, and shipping processes, they produced different silk than they naturally would. Because MaSp2 production is energetically more expensive than MaSp1 (Blamires et al., 2012b), spiders may down regulate MaSp2 expression to save energy when stressed. However, finding the ultimate cause of this pattern needs more investigation.

Although proline content can vary from day to day, the variation within a day is significantly lower than the variation among days (Figure 5). This suggests $A$. trifasciata produces nearly homogeneous silk fibers within a single day, but the composition can vary among days as well as individuals. We suggest change in relative expression rates of MaSp1 and MaSp2 to be the main - if not the only pathway in the production of silk fibers with different chemical composition within an individual of $A$. trifasciata.

Silk proline content varied in spider 25 and 62 over multiple days, while it was consistent in other individuals (Figure 4). Considering that spider 25 and 62 might be stressed suggests that the silk composition is usually consistent but spiders have the ability to change their silk composition over a short length $(60 \mathrm{~m})$ of a silk fiber. Because silk proteins are stored in silk glands waiting to be spun into silk fiber, there is a time lag between secreting and spinning a silk fiber with new chemical composition. Therefore, not only the fiber's length but the sampling time could be an important factor in assessing the natural variation. We found silk 
composition and mechanical performance were consistent among days in spiders that produced silk with more than $44 \%$ shrink capacity ( $10 \%$ proline) at the start of the experiment. Therefore, we suggest checking the mechanical behavior of major ampullate silk before starting an experiment to minimize including abnormal spiders in the study.

Many studies suggest that the amino composition of silk is variable among populations of spiders (Tso et al., 2005; Higgins et al., 2001; Craig et al., 2000). Ohio spiders showed higher proline content in major ampullate silk compared to Florida spiders (Figure 7). While this could be an evidence of geographic differences in silk genes expression, we think that it is equally likely that the Florida spiders were stressed due to collecting, storing and/or shipping processes. This stress hypothesis is supported by our observations in which some spiders from Florida produced normal silk from the first day of arrival, while others produced silk with notably unusual mechanical properties (e.g. low shrink capacity) within two days of arrival in the laboratory, but then produced silk with normal amino acid compositions and typical mechanical properties after being kept for a week in the greenhouse (unpublished data). In contrast, Ohio spiders were more consistent in amino acid composition.

Does amino acid composition correlate with silk mechanics?

We found that the proline content of major ampullate silk correlates positively with shrink capacity and true breaking strain (Figure 8). Both 
correlations are positive and plateau after 13\% proline. Other studies show this correlation between proline content and the elasticity of a fiber in general (Rauscher et al., 2006) and across different spider species (Elices et al., 2009; Liu et al., 2008b). These correlations are evidences for the hypothesis that proline increases the mobility of protein microstructure (Liu et al., 2008a; Savage and Gosline, 2008a; Rauscher et al., 2006; Hayashi and Lewis, 1998). Thus, a silk fiber with more proline has more mobile microstructures so that is more extensible and also shrinks more after supercontraction. Contrary to the other studies, proline does not correlate with breaking stress and Young's modulus in our study. Liu et al. (2008b) showed that spider silk proline content correlates with breaking stress and Young's modulus. However, the relationship is not linear and both of these correlations are strongest in the range of $0-6 \%$ proline, with the strength of the relationship declining after $7 \%$ proline. The proline contents of our samples were in the range of 5-13\% (and mostly 10-13\%). Therefore, it is possible that we did not see these correlations simply because the proline content of the silk was already high enough to put the material well beyond the amyloid-elastomer transition boundary hypothesized by Rauscher et al. (2006).

Most of the prior studies also investigate variation across different spider species (Elices et al., 2009; Liu et al., 2008b). However, these species vary not only in the relative expression of MaSp1 and MaSp2, but also in the amino acid sequences of each MaSp protein (e.g. Argiope trifasciata versus Latrodectus hesperus). We therefore conclude that the actual MaSp genes' sequences (the number of repetitive 
regions and the length of each unit) are important, in addition to their relative abundances for determining silk mechanical properties. 


\section{CHAPTER V}

\section{CONCLUSIONS}

We showed that the chemical composition of major ampullate silk is in general remarkably homogeneous within a single population of spiders at multiple spatial and temporal scales, but that chemical composition can also vary substantially across individual spiders or from day to day in ways that correlate strongly with the material properties of spider silk. We showed that the variation in chemical composition is not detectable within silk spun on a single day. However, the variation within a single spiders' silk across days can be larger than variation among individuals. Most of the variation in silk composition in our investigation results from a small number of outliers, sampled from spiders with a recent history of stress. We showed that proline is an indicator of MaSp2 and there is a strong correlation between major ampullate silk proline content and its shrink capacity after supercontraction as well as its breaking strain. This suggests that spiders are able to change the relative expression rate of different MaSp genes to produce silk fibers with different mechanical properties, which could potentially tailor the silk to function in different environments. 


\section{REFERENCES}

Agnarsson, I., Boutry, C., Wong, S.-C., Baji, A., Dhinojwala, A., Sensenig, A.T., Blackledge, T.A., 2009. Supercontraction forces in spider dragline silk depend on hydration rate. Zoology 112, 325-331.

Agnarsson, I., Kuntner, M., Blackledge, T.A., 2010. Bioprospecting finds the toughest biological material: extraordinary silk from a giant riverine orb spider. PloS one 5.

Ayoub, N.A., Garb, J.E., Hedin, M., Hayashi, C.Y., 2007a. Utility of the nuclear proteincoding gene, elongation factor-1 gamma (EF-1 gamma), for spider systematics, emphasizing family level relationships of tarantulas and their kin (Araneae : Mygalomorphae). Molecular Phylogenetics and Evolution 42, 394-409.

Ayoub, N.A., Garb, J.E., Tinghitella, R.M., Collin, M.A., Hayashi, C.Y., 2007b. Blueprint for a high-performance biomaterial: full-length spider dragline silk genes. PloS one 2, e514.

Ayoub, N.A., Hayashi, C.Y., 2008. Multiple recombining loci encode MaSp1, the primary constituent of dragline silk, in widow spiders (Latrodectus: Theridiidae). Molecular Biology and Evolution 25, 277-286.

Blackledge, T.A., 2012. Spider silk: a brief review and prospectus on research linking biomechanics and ecology in draglines and orb webs. Journal of Arachnology $40,1-12$.

Blackledge, T.A., Cardullo, R.A., Hayashi, C.Y., 2005. Polarized light microscopy, variability in spider silk diameters, and the mechanical characterization of spider silk. Invertebrate Biology 124, 165-173.

Blackledge, T.A., Hayashi, C.Y., 2006. Silken toolkits: biomechanics of silk fibers spun by the orb web spider Argiope argentata. Journal of Experimental Biology $209,2452-2461$. 
Blamires, S.J., Chao, I.C., Tso, I.M., 2010. Prey type, vibrations and handling interactively influence spider silk expression. Journal of Experimental Biology 213, 3906-3910.

Blamires, S.J., Wu, C.L., Blackledge, T.A., Tso, I.M., 2012a. Environmentally induced post-spin property changes in spider silks: influences of web type, spidroin composition and ecology. Biological Journal of the Linnean Society 106, 580588.

Blamires, S.J., Wu, C.L., Tso, I.M., 2012b. Variation in protein intake induces variation in spider silk expression. PloS one 7, e31626.

Boutry, C., Blackledge, T.A., 2010. Evolution of supercontraction in spider silk: structure-function relationship from tarantulas to orb-weavers. Journal of Experimental Biology 213, 3505-3514.

Casem, M.L., Turner, D., Houchin, K., 1999. Protein and amino acid composition of silks from the cob weaver, Latrodectus hesperus (black widow). International Journal of Biological Macromolecules 24, 103-108.

Craig, C.L., Riekel, C., Herberstein, M.E., Weber, R.S., Kaplan, D., Pierce, N.E., 2000. Evidence for diet effects on the composition of silk proteins produced by spiders. Molecular Biology and Evolution 17, 1904-1913.

Craig, V., Yang, H., Eric, G., Simon, T., Felicia, J., 2011. Spider silk composites and applications. Metal, Ceramic and Polymeric Composites for Various Uses, ISBN: 978-953-307-353-8 303-324.

Eisoldt, L., Smith, A., Scheibel, T., 2011. Decoding the secrets of spider silk. Materials Today 14, 80-86.

Elices, M., Plaza, G.R., Arnedo, M.A., Perez-Rigueiro, J., Torres, F.G., Guinea, G.V., 2009. Mechanical behavior of silk during the evolution of orb-web spinning spiders. Biomacromolecules 10, 1904-1910.

Elices, M., Plaza, G.R., Perez-Rigueiro, J., Guinea, G.V., 2011. The hidden link between supercontraction and mechanical behavior of spider silks. Journal of the Mechanical Behavior of Biomedical Materials 4, 658-669.

Garb, J.E., Ayoub, N.A., Hayashi, C.Y., 2010. Untangling spider silk evolution with spidroin terminal domains. BMC Evolutionary Biology 10, 243. 
Garb, J.E., DiMauro, T., Vo, V., Hayashi, C.Y., 2006. Silk genes support the single origin of orb webs. Science 312, 1762-1762.

Gatesy, J., Hayashi, C., Motriuk, D., Woods, J., Lewis, R., 2001. Extreme diversity, conservation, and convergence of spider silk fibroin sequences. Science 291, 2603-2605.

Hayashi, C.Y., Lewis, R.V., 1998. Evidence from flagelliform silk cDNA for the structural basis of elasticity and modular nature of spider silks. Journal of Molecular Biology 275, 773-784.

Hayashi, C.Y., Shipley, N.H., Lewis, R.V., 1999. Hypotheses that correlate the sequence, structure, and mechanical properties of spider silk proteins. International Journal of Biological Macromolecules 24, 271-275.

Higgins, L.E., Townley, M.A., Tillinghast, E.K., Rankin, M.A., 2001. Variation in the chemical composition of orb webs built by the spider Nephila clavipes (Araneae, Tetragnathidae). Journal of Arachnology 29, 82-94.

Lewis, R.V., 1992. Spider silk - the unraveling of a mystery. Accounts of Chemical Research 25, 392-398.

Liu, Y., Shao, Z., Vollrath, F., 2008a. Elasticity of spider silks. Biomacromolecules 9, 1782-1786.

Liu, Y., Sponner, A., Porter, D., Vollrath, F., 2008b. Proline and processing of spider silks. Biomacromolecules 9, 116-121.

Lombardi, S.J., Kaplan, D.L., 1990. The amino acid composition of major ampullate gland silk (dragline) of Nephila clavipes (Araneae, Tetragnathidae). Journal of Arachnology 18, 297-306.

Madsen, B., Shao, Z.Z., Vollrath, F., 1999. Variability in the mechanical properties of spider silks on three levels: interspecific, intraspecific and intraindividual. International Journal of Biological Macromolecules 24, 301-306.

Nelson, D.L., Cox, M.M., 2000. Lehninger Principles of Biochemistry. W. H. Freeman, New York.

Rauscher, S., Baud, S., Miao, M., Keeley, Fred W., Pomès, R., 2006. Proline and glycine control protein self-organization into elastomeric or amyloid fibrils.

Structure 14, 1667-1676. 
Savage, K.N., Gosline, J.M., 2008a. The effect of proline on the network structure of major ampullate silks as inferred from their mechanical and optical properties. Journal of Experimental Biology 211, 1937-1947.

Savage, K.N., Gosline, J.M., 2008b. The role of proline in the elastic mechanism of hydrated spider silks. Journal of Experimental Biology 211, 1948-1957.

Smith, B.J., 2003. Methods in Molecular Biology. Humana Press, Totowa, New Jersey

Stein, W.H., Moore, S., 1950. Chromatographic determination of the amino acid composition of proteins. Cold Spring Harbor Symposia on Quantitative Biology 14, 179-190.

Tso, I.M., Chiang, S.-Y., Blackledge, T.A., 2007. Does the giant wood spider Nephila pilipes respond to prey variation by altering web or silk properties? Ethology 113, 324-333.

Tso, I.M., Wu, H.C., Hwang, I.R., 2005. Giant wood spider Nephila pilipes alters silk protein in response to prey variation. Journal of Experimental Biology 208, 1053-1061.

Vollrath, F., Madsen, B., Shao, Z.Z., 2001. The effect of spinning conditions on the mechanics of a spider's dragline silk. Proceedings of the Royal Society BBiological Sciences 268, 2339-2346.

Work, R.W., 1977. Dimensions, birefringences, and force-elongation behavior of major and minor ampullate silk fibers from orb-web-spinning spiders effects of wetting on these properties. Textile Research Journal 47, 650-662.

Work, R.W., 1981. A comparative-study of the supercontraction of major ampullate silk fibers of orb-web-building spiders (Araneae). Journal of Arachnology 9 , 299-308.

Work, R.W., Young, C.T., 1987. The amino acid compositions of major and minor ampullate silks of certain orb-web-building spiders (Araneae, Araneidae). Journal of Arachnology 15, 65-80. 